\title{
Structural Basis for the tight binding inhibition of E coli. CTP Synthase Inhibition by Gemcitabine and its analogues
}

\author{
M McLeod ${ }^{1}$, T Holyoak ${ }^{2}$ \\ ${ }^{1}$ Biology, Univ of Waterloo, Waterloo, ${ }^{2}$ Biology Dept, Univ of Waterloo \\ mjm758@cornell.edu
}

CTP synthase (CTPS) is a vital metabolic enzyme which controls the pyrimidine nucleotide pool by converting UTP into CTP with the use of ATP and ammonia. The E coli. variant is tightly regulated by the available nucleotides, where it is allosterically modulated by GTP and CTP. CTP binding can induce inhibitory filament formation, whereas the human isozyme forms active filaments upon UTP binding. Due to the in vivo importance of CTPS, variable regulatory mechanism across species, and previous data suggesting it is vital to the maintenance of various disease states, selective inhibition of CTPS may be a fruitful avenue to develop treatments for some of these illnesses. Most recently, a series of fluorinated CTP analogues were characterized for their inhibition against CTPS. Of these molecules, a doublely fluorinated at the 2 ' position of the ribose moiety had an at least $30 \mathrm{x}$ increased inhibition over its monofluorinated counterparts. Here, we determined the crystal structures of the various fluorinated inhibitors of CTPS to determine the molecular interactions and conformational changes contributing to the potent inhibition of the enzyme by these nucleotide analogues.

Acta Cryst. (2020). A76, a81 